\title{
Gestión, planificación y control de la producción
}

Guillermo Barcelli G.

Ingeniero mecánico electricista por la Universidad Nacional de Ingeniería. Máster en banca y finanzas por la Universidad de Lima. Especialista en administración de la producción industrial,

Becado por la Carl Duisberg Gesellschaft. Consultor de empresas. Presidente de la Acep

(Asociación de Consultores Empresariales del Perú).

El objetivo fundamental de este artículo es proponer un punto de partida que permita un adecuado desarrollo de la administración de la función o sistema de producción de las empresas nacionales, en el convencimiento de su gran importancia y trascendencia como ente generador de vitales ventajas competitivas.

El autor define la gestión de operaciones y las etapas por las cuales ha atravesado. Además de presentarnos los módulos que comprende la gestión de operaciones, resalta otros temas que se deben considerar para que el gerente de producción cumpla un papel protagónico en el nuevo milenio. 
Antes se le denominaba "administración" o "dirección de la producción”, ahora se le llama "administración”, "dirección” o "gestión de operaciones"; de esta manera se pretende resaltar que se incluyen los servicios. Se le define como el conjunto de actividades que aseguran la continuidad y el funcionamiento armónico de un sistema productivo o como la administración de los recursos necesarios para producir los bienes y servicios que ofrece una organización. Se trata de la administración de la función de producción, de la obtención de productos -bienes o servicios- a partir de los recursos. Con esto nos estamos refiriendo a gerenciar el sistema de producción de la empresa que, mediante la tarea laboral, transforma la corriente de entrada en una corriente de salida, es decir, se intenta dirigir los procesos de transformación que agregan valor.

También hay variaciones y diferencias en cuanto a su contenido. Algunos autores mencionan que éste debe adecuarse al entorno y que la dirección de operaciones, aunque las utiliza, no es la ingeniería industrial con sus métodos analíticos, tareas y procesos, ni el riguroso trato matemático de la investigación operativa. Ambas tienen un enfoque técnico y académico y, en la práctica, proporcionaron a las empresas resultados que fueron ensombrecidos por los métodos de gestión japoneses. Éstos, partiendo de ideas occidentales, llegaron a establecer conceptos, técnicas e instrumentos prácticos, empleando métodos deductivos con un enfoque de largo plazo, logrando conformar y establecer una filosofía que, desde hace ya varios años, es seguida por los países occidentales.

Otros autores definen las "operaciones" como un término más amplio que "producción", considerando que incluye todas las actividades de la cadena de valor, desde el proveedor hasta el cliente. Es decir, comprende las actividades del abastecimiento de insumos (adquisición y almacenamiento de materiales), de la gestión de los procesos productivos, y del almacenamiento y distribución de los productos terminados.

La administración de la producción ha pasado por varias etapas, jalonadas por conceptos tales como la mecanización (James Watt, 1764), la división del trabajo (Adam Smith, 1776), la dirección científica y el estudio del trabajo (Frederick Taylor, 1911), la estandarización de partes (Henry Ford, 1913), los modelos matemáticos de decisión (F.W. Harris, 1915), las relaciones humanas (Elton Mayo, 1930) y la investigación de operaciones (George Dantzig, 1947). En los últimos 50 años la 
gestión de operaciones ha sido modelada por el empleo de la computación, la atención enfocada en los procesos, la gestión participativa, la preponderancia de la calidad y la productividad, la gestión basada en el tiempo, la robótica, los sistemas de manufactura flexible, la manufactura integrada por computadora, y por la liberalización y globalización de los mercados.

En los años setenta, aprovechando el rápido desarrollo de la computación se realizó en Estados Unidos la denominada "cruzada MRP”, iniciándose con la planificación de los requerimientos de materiales (MRP I), para pasar a la planificación de los recursos de manufactura (MRP II), luego a la planificación de los recursos de gestión (MRP III), a la planificación de los recursos de distribución (DRP), y, por último, ahora se menciona la planificación de los recursos de la empresa (ERP).

En los años ochenta se divulgó lo que significó una revolución de la filosofía de la producción, el justo-a-tiempo o Just-inTime (JIT), que junto con el TQM y el TPM conforman un sistema para el mejoramiento continuo de la calidad y productividad a través de la participación (Kaizen).

Actualmente, en el cambio de siglo, la producción está retomando el papel protagónico que le corresponde, se está tomando conciencia de la importancia de su función agregadora de valor y de su capacidad para proporcionar ventajas competitivas. Se está volviendo a apreciar el impacto que tiene en la competitividad al incidir en la calidad, costos, servicios, despachos e interacción con los clientes.

Como consecuencia de toda esta evolución, de los cambios de paradigmas y de la adecuación a las nuevas exigencias del entorno, en la actualidad se tiende a considerar que la gestión de operaciones comprende los siguientes módulos:

\begin{tabular}{ll}
\hline Módulo & Objetivos \\
\hline Análisis de los procesos & Proporcionar una estructura analítica a la función \\
Modelación de la cadena de suministro & Estipular modelos para el análisis de la cadena \\
Sistemas de producción de clase mundial & Responder a las exigencias del entorno \\
Estrategias de operaciones & Proporcionar algunos cursos de acción alternativos \\
Gestión internacional o global de operaciones & $\begin{array}{l}\text { Administrar una producción internacional } \\
\text { Integración interfuncional con otras áreas }\end{array}$ \\
& $\begin{array}{l}\text { Integrar las funciones de la empresa con la produc- } \\
\text { ción }\end{array}$ \\
\hline
\end{tabular}


El resultado de las investigaciones realizadas en Estados Unidos y Europa, para conocer el contenido que actualmente tienen los sílabos de Administración de la Producción o Gestión de Operaciones, permite concluir que sus principales universidades han estructurado este curso de acuerdo con esos módulos, enfatizando aquél que, según especiales circunstancias, lo amerite. También se concluye de dichas investigaciones que en los cursos de pregrado, donde los alumnos no tienen experiencia todavía, la metodología empleada se basa en la exposición teórica, y que en los cursos de posgrado, aprovechando la experiencia que se exige a los participantes, la metodología descansa (un promedio de 64\% de la duración de los cursos, variando entre 50\% y 88\% de las sesiones) en la solución de casos.

Se ha definido que el gerente de producción del tercer milenio tendrá que integrar personas, materiales, medios de elaboración, información y tiempo, que debe ser capaz de emplear en forma combinada enfoques tecnológicos, económicos, organizativos y de gestión, para analizar, planificar y dirigir sistemas industriales complejos. El gerente de producción interviene en todas las fases operacionales de la cadena de valor-diseño, compra, producción y distribución- participando en el diseño de sistemas productivos sociotécnico-económicos, en el planeamiento de la producción y la gestión de materiales, en el análisis del valor y la selección de la tecnología, en el mantenimiento y la calidad, en la contabilidad y finanzas, y en la estipulación de las estrategias del negocio y de las operaciones.

Por lo tanto, el gerente de producción debe ser un experto en sistemas, tecnología, organización y gestión, con amplios conocimientos de ingeniería, proyectos de innovación tecnológica, logística, economía, márketing, dirección de personal, finanzas, y evaluación y manejo del riesgo.

Para proporcionar el conocimiento integral que se exige, en cada uno de los módulos antes mencionados se debe incluir temas como los que se presentan a continuación en una lista que intenta recoger, de manera natural, sin mayor orden ni clasificación, la dispersa información que existe en la actualidad.

\section{Análisis de procesos}

Proporciona una estructura analítica para la administración de la producción como una función del negocio, incluye la 
medición del desempeño de las operaciones, permite seleccionar el proceso adecuado para la estrategia de negocio planteada según los requerimientos del entorno.

\section{Temas}

Calidad

Teoría estadística para control de calidad

Productividad

Costo

Flexibilidad

Servicio

Gestión de procesos

Diseño de procesos

Análisis de procesos

Investigación de operaciones

Técnicas cuantitativas de modelación

Secuenciación

Diseño del trabajo

Medición del trabajo

Control de operaciones

Sistemas de medición de desempeños

MRP (planeación de recursos de manufactura)

JIT (justo a tiempo)

Tecnología

Ingeniería

Mantenimiento

Relaciones laborales

Modelación de la cadena de suministro

Estipula modelos para el análisis de la cadena de suministro, estudia la relación proveedor-cliente, introduce los métodos cuantitativos tradicionales, refuerza el enfoque de procesos de los componentes de la cadena de suministro.

\section{Temas}

Gestión de la cadena de suministro

Estrategias de la cadena de suministro 
Componentes y entidades de una cadena de suministro

Desempeño de la cadena de suministro

Compras

Modelos de lote económico

Gestión de inventarios

MRP I (planificación de los requerimientos de materiales)

JIT (justo a tiempo)

ERP (planeación de los recursos de la empresa)

Outsourcing

Técnicas de presupuestación

Planeamiento y control de la producción

Planeamiento agregado

Planeamiento de la capacidad

Teoría de programación

Programación

Localización de recursos

EDI (intercambio electrónico de datos)

Internet

Sistemas de producción de clase mundial

Responde al entorno competitivo cambiante, articula estrategias con la práctica, proporciona una apreciación de las mejores prácticas actuales para la gestión de las operaciones.

\section{Temas}

Competitividad

Gestión estratégica

Gestión del suministro

Gestión de servicios

Manufactura de clase mundial

Prácticas de operaciones

Kaizen

TQM (gestión de calidad total)

JIT (justo a tiempo)

TPM (mantenimiento productivo total)

TQC (control total de la calidad)

Sistemas de manufactura celular

Círculos de calidad

TOC (teoría de restricciones)

CAD (diseño ayudado por computadora) 
CAE (ingeniería ayudada por computadora)

CAM (manufactura ayudada por computadora)

CAPP (planeamiento de producción ayudado por computadora)

Innovación

FMS (sistemas de manufactura flexible)

CIM (manufactura integrada por computadora)

Trabajo en equipo

Motivación

Sistemas de sugerencias

Reingeniería

Ingeniería concurrente

Ecología

Estrategias de operaciones

Incluye aspectos amplios sobre los cursos de acción alternativos para tomar las grandes decisiones, establece pautas para equilibrar la demanda con las capacidades internas.

\section{Temas}

Desarrollo de producción nueva

Diseño de sistemas de producción

Estrategias de productos

Estrategias de procesos

Estrategia de tecnología

Elección de la tecnología

\section{Gestión internacional o global de operaciones}

Atiende las necesidades de aprender a gerenciar operaciones distribuidas en varios países y de comprender las sutilezas de la globalización y las exigencias de la liberalización para redefinir las ventajas competitivas.

\section{Temas}

Operaciones distribuidas internacionalmente

Localización de planta

Aspectos culturales

Aspectos organizacionales 
Productividad en un contexto global

Sistemas de información

Tecnología de las informaciones y comunicaciones

Estrategias de tecnología

Transferencia de tecnología

Automatización

EDI (intercambio electrónico de datos)

Estrategias de investigación y desarrollo

Manufactura internacional

Márketing global

Distribución global

Gestión de la cadena de suministro

Cadenas de suministro globales

Operaciones de servicio globales

Integración interdisciplinaria con otras áreas funcionales

Desarrollo continuo de la integración con tópicos que tradicionalmente se hallan en otras áreas, hace menos nítidos sus límites funcionales, dinamiza la administración de la producción y repotencia la empresa a través de la sinergia que logra.

\section{Temas}

Sistemas de información

Mercadotecnia

Finanzas

Logística

Organización

Gestión de servicios

Localización de la planta

Distribución de la planta

Gestión de la cadena de suministro

Desarrollo de producción nueva

Gestión ambiental

Tecnologías limpias

Impacto ecológico

Gestión de proyectos

Conducta organizacional

Gestión del cambio

Procesos de negocio y diseños de sistemas

Reingeniería 
Gestión de la renta

Organizaciones de respuesta sensible rápida

Logística de respuesta rápida

Gestión de respuesta rápida

Sistema de medición de desempeño

Pequeñas y medianas empresas

Consultoría empresarial

Empresariado

Se entiende que esta relación no es limitativa, y que se debe actualizar con la frecuencia que exige un entorno cambiante y exigente. También se observa que algunos temas figuran en más de un módulo; además, que ciertos temas están comprendidos en otros de los señalados, que algunos son generales y otros, más específicos, son parte de aquéllos.

Dentro de la gestión de la producción desempeña un rol importante la planificación y conducción operativa de la producción, denominada también "planificación" y "control de producción”.

La planificación y control de la producción comprende los procedimientos y la información que se utiliza para lograr que funcione eficaz y eficientemente el sistema productivo y sus procesos de transformación. Es la dirección y coordinación de recursos para alcanzar los objetivos de producción predeterminados con la máxima rentabilidad posible. También ha sido definida como el sistema de información que proporciona el soporte para la administración eficiente de los recursos -personal, materiales y medios de elaboración- y la coordinación de las actividades internas con las de proveedores y clientes.

La planificación y control de producción se puede estructurar con un enfoque jerárquico, que, basado en los diferentes niveles de tiempo, propondría planificaciones de la producción a largo, mediano y corto plazo:

\section{Planificación de la producción a largo plazo}

Planificación estratégica de los tipos de productos Planificación de la capacidad a largo plazo

\section{Planificación de la producción a mediano plazo}

Planificación agregada de las familias de productos Planificación agregada de la capacidad 
Planificación de la producción a corto plazo

Programación maestra de los productos

Planes de trabajo de los productos

Plan aproximado de la capacidad

Planificación de los materiales

\section{Planificación del taller}

Programación de los componentes

Conducción operativa de la producción

Plan detallado de la capacidad

Conducción operativa de los materiales

Debe tenerse presente que después de la planificación del taller, es decir en el muy corto plazo, lo que sigue es la ejecución de los trabajos, que exige capacidades y recursos como:

Personal

Información

Conocimiento

Experiencia

Medios de elaboración

Tecnología

La planificación y conducción operativa de la producción debe comprender temas como:

Expansión de la capacidad

Contracción de la capacidad

Toma de decisiones

Desarrollo de procesos

Desarrollo de productos

Innovación

Tecnología

Planificación financiera

Análisis financiero de la producción

Pronósticos

Plan agregado de la capacidad

Plan agregado de la producción

Listas de capacidad

Perfiles de recursos

Planificación agregada de recursos

Programa maestro de producción

Lista de piezas (gozinto)

Prioridad 


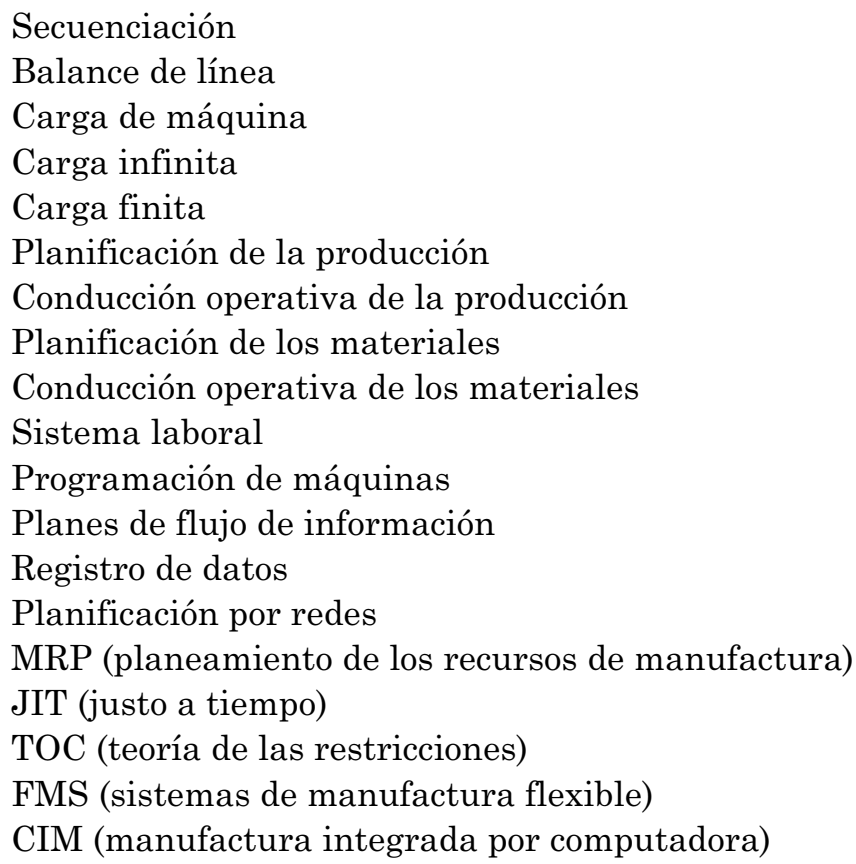

Se debe tener presente que esta lista es indicativa, no limitativa, y que ciertos temas han sido mencionados en forma más específica que otros, además, que algunos temas podrían estar comprendidos en otros.

Con este artículo se está intentando iniciar una adecuada administración de la producción en el país. Si bien es cierto que se debe clasificar y ordenar los temas expuestos, también es verdad que se deberá diseñar los cursos de pregrado y posgrado sobre gestión de operaciones considerando la cobertura e interrelaciones de cada uno de ellos; se trata de obtener un currículo moderno, y, sobre todo, debidamente coordinado y adecuado a las necesidades de la industria nacional.

Otro objetivo de este artículo es plantear un reto a todos aquéllos que, fascinados por la producción y conscientes de su importancia, sienten la responsabilidad de hacer un modesto aporte a la producción nacional, requisito indispensable para mejorar el nivel y la calidad de vida de todos los peruanos. Estamos seguros de que, superando las limitaciones de este artículo, lograremos desarrollar y articular educación y práctica, aulas y talleres, ciencia y tecnología. 


\section{Bibliografía}

Barcelli Gómez, Guillermo

Productividad. Lima: Centro de Investigaciones Sociales, Económicas y Tecnológicas, 1995.

Baumgartner, Horst; Klaus Knischewski y Harald Wieding

CIM. Consideraciones básicas. Madrid: Siemens Aktiengesellschaft; Berlín y Munich \& Marcombo S.A., 1991.

Companys Pascual, Ramón

Planificación y programación de la producción. Madrid: Marcombo S.A., 1989.

Companys Pascual, Ramón y Joan B. Fonollosa y Guardiet

Nuevas técnicas de gestión de stocks: MRP y JIT.

Madrid: Marcombo S.A., 1989.

Chase, Richard B. y Nicholas J. Aquilano

Dirección y administración de la producción y de las operaciones. México: Doyma Mexicana, S.A. de C.V., 1998.

Domínguez Machuca, José Antonio; María José Álvarez Gil, Santiago García González; Miguel Ángel Domínguez Machuca y Antonio Ruiz Jiménez

Dirección de operaciones. Aspectos estratégicos en la producción y los servicios. Madrid: McGraw-Hill / Interamericana de España S.A., 1995.

Dirección de operaciones. Aspectos tácticos y operativos en la producción y los servicios. Madrid: McGrawHill / Interamericana de España S.A., 1995.

Drucker, Peter

Gerencia para el futuro. Bogotá: Editorial Norma S.A., 1992.

Eilon, Samuel

La producción. Planificación, organización y control.

Madrid: Editorial Labor S.A., 1980.

Ferré Masip, Rafael

La fábrica flexible. España: Marcombo S.A., 1988. 
Goldratt, Eliyahu M. y Jeff Cox

La meta. México: Ediciones Castillo, S.A. de C.V., 1995.

Hirano, Hiroyuki

El JIT, revolución en las fábricas, una guía gráfica para el diseño de la fábrica del futuro. Madrid: Tecnologías de Gerencia y Producción S.A., 1992.

Imai, Masaaki

Cómo implementar el kaizen en el sitio de trabajo (gemba). Bogotá: McGraw-Hill / Interamericana, S.A., 1998.

International Journal of Operations \& Production Management London: MCB University Press, 1989-1999.

Monks, Joseph G.

Administración de operaciones. México: McGraw-Hill / Interamericana de México, S.A. de C.V., 1993.

Narasimhan, Seetharama L.; Dennis W. McLeavey y Peter J. Billington

Planeación de la producción y control de inventarios. México: Prentice Hall Hispanoamericana, S.A., 1996.

Noori, Hamid y Russell Radford

Administración de operaciones y producción. Calidad total y respuesta sensible rápida. Colombia: McGrawHill / Interamericana S.A., 1997.

Plossl, George W.

Control de la producción y de inventarios, principios y técnicas. México: Prentice Hall Hispanoamericana, S.A., 1987.

Schonberger, Richard J.

Manufactura de categoría mundial. Bogotá: Editorial Norma S.A., 1989.

Técnicas japonesas de fabricación. México: Editorial Limusa S.A. de C.V., 1993.

Schroeder, Roger G.

Administración de operaciones. Toma de decisiones en la función operaciones. México: McGraw-Hill / Interamericana de México, S.A. de C.V., 1990. 
Shingo, Shigeo

Producción sin stocks: el sistema Shingo para la mejora continua. Madrid: Tecnologías de Gerencia y Producción S.A., 1991.

Vollmann, Thomas; L. William L. Berry y D. Clay Whybark Sistemas de planificación y control de la fabricación. Bogotá: Addison-Wesley Iberoamericana, S.A. / Times Mirror de España S.A., 1997. 